\title{
High prevalence of asymptomatic peptic ulcers diagnosed during screening endoscopy in patients with cirrhosis
}

\author{
Theodoros Voulgaris, Dimitrios Karagiannakis, Spyridon Siakavellas, Despina Kalogera, \\ Theodoros Angelopoulos, Elissavet Chloupi, George Karamanolis, George Papatheodoridis, \\ John Vlachogiannakos
}

LAIKO General Hospital, University of Athens, Medical School, Athens, Greece

Abstract

\begin{abstract}
Background Peptic ulcer disease (PUD) is more prevalent in cirrhotics and this may aggravate prognosis. We investigated the prevalence of PUD in cirrhotics and its potential association with Helicobacter pylori (H. pylori) infection, the underlying etiology and severity of liver disease, and other manifestations of portal hypertension $(\mathrm{PH})$.
\end{abstract}

Methods We enrolled consecutive asymptomatic cirrhotic patients who underwent screening endoscopy in a tertiary hospital during a 12-month period. We recorded the presence of PUD and the endoscopic findings associated with $\mathrm{PH}$. H. pylori infection was documented through either histology or CLO-test. The diagnosis of cirrhosis was based on elastography, liver biopsy or a combination of clinical, biochemical and imaging data.

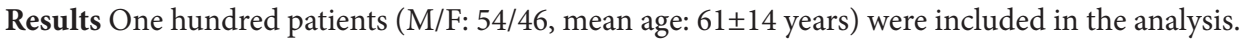
Viral hepatitis (37\%) and alcohol (22\%) were the most common causes of cirrhosis. Child-Pugh stage was A/B/C: 60/35/5. PUD was found in 19 patients (14 gastric, 5 duodenal). H. pylori infection was diagnosed in 54\%. Varices were detected in 59\% (39\% needed treatment). PH gastropathy was present in $81 \%$ (severe in $33 \%$ ). The presence of PUD was unrelated to the etiology and the severity of liver disease or to other endoscopic manifestations of $\mathrm{PH}$. No correlation was found between PUD and $H$. pylori infection.

Conclusions A high prevalence of PUD was observed in our cirrhotic patients, although they were asymptomatic and had no known risk factors of ulcerogenicity. The value of screening endoscopy for the early diagnosis and treatment of PUD in cirrhotics deserves further investigation.

Keywords Liver cirrhosis, peptic ulcer disease, Helicobacter pylori infection, portal hypertension Ann Gastroenterol 2019; 32 (4): 1-6

\section{Introduction}

Patients with cirrhosis can develop multiple complications as a consequence of portal hypertension and liver decompensation [1]. Gastrointestinal (GI) bleeding is a lifethreatening event observed frequently in cirrhosis and is

Academic Department of Gastroenterology and Hepatology, LAIKO General Hospital, University of Athens, Medical School, Athens, Greece

\section{Conflict of Interest: None}

Correspondence to: John Vlachogiannakos, MD, Department of Gastroenterology, Medical School of National and Kapodistrian University of Athens, Laiko General Hospital, Agiou Thoma 17, 11527 Athens, Greece, e-mail: jvlachog@hotmail.com

Received 3 February 2019; accepted 5 May 2019; published online 6 July 2019

DOI: https://doi.org/10.20524/aog.2019.0399 commonly attributed to variceal bleeding [2]. Since nonbleeding varices are asymptomatic, screening upper GI endoscopy is mandatory for cirrhotic patients in order to assess the indication for prophylactic treatment [3]. However, upper GI bleeding (UGIB) in cirrhosis is not synonymous with variceal bleeding. Several studies have reported that peptic ulcer can be the cause of bleeding in almost $10 \%$ of cirrhotics who present with UGIB $[2,4]$.

Previous studies have pointed out the high prevalence of peptic ulcer disease in patients with cirrhosis [5-8]. Furthermore, it has been shown that such patients have a significantly higher risk of peptic ulcer bleeding compared with the general population [9]. Moreover, there are data underlining that any episode of UGIB due to peptic ulcer worsens the prognosis of cirrhotic patients $[10,11]$. In a recent study, the 90-day mortality of cirrhotic patients after peptic ulcer bleeding was $25 \%$, significantly higher than that of noncirrhotic patients [12]. More recently, in a multicenter study, the authors concluded that patients with cirrhosis and acute 
peptic ulcer bleeding have a 45 -day mortality risk similar to that of patients who bleed from varices, further emphasizing the importance of peptic ulcer disease in this setting [13]. However, the existing data have limitations, as many of the published studies were retrospective and did not assess the presence of Helicobacter pylori (H. pylori) infection. Moreover, most of the studies included only patients with symptomatic GI bleeding.

The exact pathophysiology of ulcerogenesis in cirrhotic patients has not been fully elucidated, as there are conflicting data concerning the contribution of $H$. pylori infection and the potential role of portal hypertension $[5,7,8,14,15]$. In the present study, we prospectively evaluated the prevalence and risk factors of peptic ulcer in asymptomatic cirrhotic patients who underwent screening upper GI endoscopy.

\section{Patients and methods}

\section{Patients}

Consecutive patients with cirrhosis who attended the outpatient liver clinics of our Department during a 12-month period were invited to participate in the study. Patients were considered eligible if they were over 18 years of age and fulfilled the criteria for screening upper GI endoscopy, either on the basis of an initial diagnosis of cirrhosis or during follow up for gastroesophageal varices and within the guidelines established by the Baveno VI consensus workshop [2]. We excluded patients with acute on chronic liver failure (ACLF), fulminant hepatic failure, known hepatocellular carcinoma (HCC) or other extrahepatic malignancies, transjugular intrahepatic portosystemic shunts (TIPS), previous surgical treatment for portal hypertension, and a history of gastric surgery or known peptic ulcer disease. We also excluded patients who had received antacids, $\mathrm{H}_{2}$-receptor antagonists, proton pump inhibitors, or antiplatelet, anticoagulation or anti-inflammatory agents during the last month before upper GI endoscopy.

The study protocol conformed to the principles outlined in the Declaration of Helsinki and was approved by the Ethics Committee of the hospital. All participants gave written informed consent.

The diagnosis of cirrhosis was based on histological and/ or elastographic findings for patients with compensated liver disease and on clinical and/or radiological findings for patients with decompensated liver disease. Based on reliable liver elastography, patients with compensated liver disease were considered to have cirrhosis if they were found to have liver stiffness $>14 \mathrm{kPa}$. The clinical and/or radiological diagnosis of cirrhosis with decompensated liver disease was made in cases with chronic liver disease of any cause and current or history of ascites, variceal bleeding, encephalopathy, and/ or non-obstructive jaundice. The severity of cirrhosis was assessed using the Child-Pugh classification [16]. Adopting the Baveno VI consensus workshop statements, supported by more recently published data, patients with liver stiffness by elastography $<20 \mathrm{kPa}$ and platelet (PLT) counts $>150,000 / \mathrm{mm}^{3}$ were considered to be most unlikely to have clinically significant portal hypertension (CSPH) $[3,17,18]$.

\section{Endoscopy}

All patients included in the study underwent upper GI endoscopy. Gastroduodenal lesions were classified as gastric ulcer, duodenal ulcer, portal hypertensive gastropathy (PHG), esophageal varices, gastric varices, or no abnormal findings. Active ulcer was defined as a fibrin-covered mucosal lesion with a minimal size of $5 \mathrm{~mm}$. The presence and severity of PHG were defined according to the North Italian Endoscopic Club classification [19], while varices identified endoscopically were classified as either small or medium/large, with the cutoff diameter being $5 \mathrm{~mm}$ [20]. Varices were further classified as having a low (LRV) or high risk (HRV) for bleeding. LRV included small varices, while HRV represented small varices with red color signs or medium/large varices [3].

At each endoscopy, a total of 5 biopsy specimens were taken with sterilized biopsy forceps ( 3 from the antral mucosa proximal to the pylorus and 2 from the greater curvature of the corpus). Two samples from the antrum and 2 samples from the body of the stomach were fixed in $10 \%$ formalin and sent for histopathologic examination (Giemsa staining). The remaining specimen from the antrum was used for the rapid urease analysis (CLO-test, Delta West Ltd., Australia). Patients were considered to have $H$. pylori infection if $H$. pylori was detected by either the histopathologic examination or the rapid urease test.

All endoscopies were performed by 2 endoscopists (JV, GP) with more than 3 years' experience. On the day of endoscopy, a complete medical history was obtained from each patient and all recent (within the previous 3 months) liver-related laboratory tests, imaging, and elastography results were recorded.

\section{Statistical analysis}

Values are expressed as mean $\pm \mathrm{SD}$ or median (range). Quantitative variables were compared between patients with or without peptic ulcer using the sample Student's $t$-test or the Mann-Whitney $U$ test. Categorical data were analyzed using the $\chi^{2}$-test with Yate's correction or the 2-sided Fisher's exact test as appropriate. All statistical analyses were performed using SPSS 22.0 for Windows (SPSS, Chicago, IL, USA). A P-value $<0.05$ was considered statistically significant.

\section{Results}

\section{Patient characteristics}

From March 2017 to February 2018, 105 consecutive cirrhotic patients underwent screening upper GI endoscopy 
and were invited to participate in the study. Of these, 5 were excluded because of ACLF $(n=1)$, HCC $(n=3)$, or TIPS $(n=1)$. Finally, a total of 100 cirrhotic patients were included in this study. Viral hepatitis and alcohol abuse were the most common causes of chronic liver disease. The majority of patients were Child class A. However, half of them had a history of liver decompensation. Using the Baveno VI criteria as a discriminative tool for identifying cases without CSPH, $12 / 87$ patients $(14 \%)$ had both liver stiffness $<20 \mathrm{kPa}$ and PLT counts $>150,000 / \mathrm{mm}^{3}$. The clinical and laboratory baseline characteristics of the patients are presented in Table 1.

\section{Endoscopic findings}

Esophageal varices were found in 59/100 (59\%) patients. Of these, 39 (39\%) were considered to have a high risk for bleeding. Gastric varices were found in only 3 (3\%) patients. The majority of patients (81\%) had PHG, while severe PHG was present in $33 \%(33 / 100)$.

Peptic ulcer was detected in 19\% (19/100) of patients. Gastric ulcers were more common than duodenal ulcers (14\% or $14 / 100$ vs. $5 \%$ or $5 / 100, P=0.033)$. All gastric ulcers were observed in the antrum. Peptic ulcer was diagnosed in $14 \%$ (7/50) of patients with decompensated cirrhosis compared to $24 \%(12 / 50)$ of patients with compensated disease $(\mathrm{P}=0.202)$. In addition, peptic ulcer was detected in 23\% (14/60) of patients with Child class A, $11 \%$ (4/35) of patients with Child class B, and 20\% (1/5) of patients with Child class C $(\mathrm{P}=0.361)$.

Table 1 Baseline characteristics of 100 asymptomatic patients with cirrhosis

\begin{tabular}{lc}
\hline Characteristics & Value \\
\hline Sex $(\mathrm{M} / \mathrm{F}), \mathrm{n}$ & $54 / 46$ \\
\hline Age, years & $61 \pm 14$ \\
\hline Etiology of cirrhosis: viral/alcoholic/other, \% & $37 / 22 / 41$ \\
Child-Pugh class (A/B/C), n & $60 / 35 / 5$ \\
\hline Child-Pugh score & $6.6 \pm 1.5$ \\
\hline MELD score & $10.7 \pm 3.1$ \\
\hline History of ascites, \% & 44 \\
\hline History of encephalopathy, \% & 23 \\
\hline Bilirubin, $\mathrm{mg} / \mathrm{dL}$ & $1.5 \pm 1.1$ \\
\hline International normalized ratio & $1.3 \pm 0.2$ \\
\hline Albumin, $\mathrm{g} / \mathrm{dL}$ & $3.6 \pm 0.6$ \\
Creatinine, $\mathrm{mg} / \mathrm{dL}$ & $0.9 \pm 0.6$ \\
\hline Hemoglobin, g/dL & $12.2 \pm 2.0$ \\
\hline Platelets, $\times 10^{3} / \mathrm{mm}{ }^{3}$ & $114 \pm 51$ \\
\hline Liver stiffness, $\mathrm{kPa}$ & $24.1 \pm 9.6$ \\
\hline Use of $\beta-$ blockers, $\mathrm{n}$ & 43 \\
\hline
\end{tabular}

Quantitative variables are expressed as mean \pm standard deviation values $M E L D$, model for end-stage liver disease
Liver disease etiology was not associated with the presence of peptic ulcer. Patients with alcohol-related liver disease tended to have a greater prevalence of gastric ulcers compared to all other etiologies of cirrhosis, but the difference did not reach statistical significance $(27.3 \%$ or $6 / 22$ vs. $10.4 \%$ or $8 / 78$, $\mathrm{P}=0.076$ ). The prevalence of peptic ulcer was not associated with the presence and severity of varices, PHG, or the possibility of CSPH according to the Baveno VI criteria. However, there was a trend for a higher prevalence of gastric ulcer in patients with severe rather than mild/moderate PHG $(21.0 \%$ or $7 / 33$ vs. $8.3 \%$ or $4 / 48, \mathrm{P}=0.112$ ). Furthermore, the presence of peptic ulcer was not associated with age, sex, Child-Pugh score, model for end-stage liver disease (MELD) score, or with the complications of cirrhosis (hepatic encephalopathy, ascites), levels of liver stiffness or liver function tests (Table 2).

Finally, the prevalence of peptic ulcer disease was $25 \%$ $(3 / 12)$ in patients most likely not to have CSPH (Baveno VI criteria), and $20 \%(15 / 75)$ in the remainder of our patient population $(\mathrm{P}=0.707)$.

\section{H. pylori infection}

Fifty-four percent of our patients were found to be positive for $H$. pylori infection. The presence of $H$. pylori infection was not correlated with the presence of peptic ulcer disease: $H$. pylori positive: $52.6 \%$ (9/19) of patients with and 54.3\% (44/81) of patients without peptic ulcer $(\mathrm{P}=0.998)$. In contrast, patients with decompensated compared to those with compensated liver disease were more commonly infected with $H$. pylori $(66 \%$ or $33 / 50$ vs. $42 \%$ or $21 / 50, \mathrm{P}=0.027)$. H. pylori infection was not associated with the presence of PHG (H. pylori positive: $54.5 \%$ [44/81] of patients with and $45.5 \%$ [9/19] of patients without $\mathrm{PHG}, \mathrm{P}=0.633$ ) or the presence of gastroesophageal varices (H. pylori positive: $52.5 \%$ [31/59] of patients with and $56.1 \%$ [23/41] of patients without varices, $\mathrm{P}=0.839$ ).

\section{Discussion}

In our prospective study, a relatively high $(\sim 20 \%)$ prevalence of peptic ulcer was found in asymptomatic patients with cirrhosis who underwent upper GI endoscopy for screening purposes. Previous studies have also reported equally high prevalences of peptic ulcer in cirrhotic patients, with rates ranging from $15-20 \%[5,6,8,21-23]$; however, most of those studies had specific limitations, mostly involving patients with additional risk factors for peptic ulcer. In particular, Bang et al revealed an ulcer prevalence of $18 \%$ among cirrhotics, but their population consisted specifically of alcoholics and $4.9 \%$ were either on non-steroidal anti-inflammatory drugs (NSAIDs) or on antiplatelet medication [21]. In an Italian study, Siringo et al found a point prevalence of peptic ulcer of $15 \%$, but $30 \%$ of their patients underwent upper GI endoscopy because of symptoms [6]. Likewise, in another Greek study by Tzathas et al, the prevalence of peptic ulcer among cirrhotics was $18 \%$, 
Table 2 Association of peptic ulcer disease with clinical, laboratory and endoscopic findings

\begin{tabular}{|c|c|c|c|}
\hline Characteristics & Peptic ulcer $(\mathrm{n}=19)$ & No peptic ulcer $(\mathrm{n}=81)$ & P-value \\
\hline $\operatorname{Sex}(M / F), n / n$ & $13 / 6$ & $46 / 35$ & 0.441 \\
\hline Age, years & $65 \pm 11$ & $60 \pm 14$ & 0.173 \\
\hline Liver disease etiology: viral/alcoholic/other, \% & $37 / 20 / 43$ & $37 / 31.5 / 31.5$ & 0.476 \\
\hline Child-Pugh class (A/B), \% & $73.7 / 26.3$ & $60.5 / 39.5$ & 0.426 \\
\hline Child-Pugh score & $6.4 \pm 1.4$ & $6.6 \pm 1.6$ & 0.262 \\
\hline MELD score & $10.9 \pm 3.1$ & $10.2 \pm 3.3$ & 0.929 \\
\hline Ascites, $\mathrm{n}(\%)$ & $6(31.6)$ & $37(46)$ & 0.307 \\
\hline Hepatic encephalopathy, n (\%) & $3(15.8)$ & $20(24.7)$ & 0.548 \\
\hline Esophageal varices n (\%) & $9(47.4)$ & $48(59.2)$ & 0.440 \\
\hline Portal hypertensive gastropathy, n (\%) & $16(84.2)$ & $64(78.9)$ & 0.756 \\
\hline Use of $\beta$-blocker, n (\%) & $8(42)$ & $35(43.4)$ & 0.999 \\
\hline Liver stiffness, $\mathrm{kPa}$ & $24.1 \pm 8.1$ & $24.3 \pm 10.2$ & 0.954 \\
\hline Baveno VI criteria for no upper GI endoscopy, n/N (\%) & $3 / 18(16,7)$ & $9 / 69(13)$ & 0.707 \\
\hline Platelets, $\times 10^{3} / \mathrm{mm}^{3}$ & $136 \pm 57$ & $109 \pm 49$ & 0.093 \\
\hline Hemoglobin, g/dL & $12.6 \pm 2.2$ & $12.1 \pm 1.9$ & 0.279 \\
\hline Bilirubin, $\mathrm{mg} / \mathrm{dL}$ & $1.2 \pm 0.6$ & $1.6 \pm 1.2$ & 0.186 \\
\hline Albumin, g/dL & $3.6 \pm 0.7$ & $3.7 \pm 0.7$ & 0.437 \\
\hline International normalized ratio & $1.2 \pm 0.2$ & $1.3 \pm 0.2$ & 0.099 \\
\hline Creatinine, $\mathrm{mg} / \mathrm{dL}$ & $1.1 \pm 1.3$ & $0.8 \pm 0.3$ & 0.359 \\
\hline
\end{tabular}

Quantitative variables are expressed as mean \pm standard deviation values

$M E L D$, model for end-stage liver disease

but they also included both symptomatic and asymptomatic patients as well as patients receiving NSAIDs or antiplatelet drugs [23]. In contrast, we established a high incidence of peptic ulcer in patients with cirrhosis encompassing all etiologies of liver disease and without any symptom, while we excluded all patients receiving drugs that predispose to peptic ulcer.

The majority of peptic ulcers observed in our study were located in the stomach, in agreement with previous reports [5,7]. Although drugs are considered the main predisposing factor for the development of gastric ulcers, obviously this was not the case in our study. Our findings are supported by those of Luo et al, who reported that the risk of developing peptic ulcer disease and/or bleeding remains higher after adjustments for ulcerogenic medications (e.g., NSAIDs, antiplatelets, anticoagulants) [9].

The fact that in all patients with gastric ulcer the ulcer was observed in the antrum and not in the body, apart from the ulcer's size, also distinguishes them from the gastric erosions commonly observed in portal gastropathy. As is well known, portal gastropathy affects mainly the body of the stomach and not the antrum [24]. Consequently, severe portal gastropathy that can lead to gastric erosions is possibly not the main pathophysiology factor that could to some extent explain the high incidence of gastric ulcers in cirrhotic patients.

H. pylori infection is an established cause of peptic ulcer in non-cirrhotic patients; hence, many studies have tried, though not successfully, to establish an association between peptic ulcer and $H$. pylori infection in cirrhotics $[6,8,25]$. We were also not able to demonstrate such an association, as the prevalence of $H$. pylori infection was not statistically different in our cirrhotic patients with or without a peptic ulcer. Only Calvet et al reported a positive correlation between $H$. pylori infection and peptic ulcer in cirrhotics [22], but the diagnosis of $H$. pylori infection was made by serological methods, which have been shown to be suboptimal in patients with cirrhosis [8]. In previous studies, the prevalence of $H$. pylori infection in cirrhotics ranged from 20-60\% [8,25-27]. We also observed a high prevalence of $H$. pylori infection among cirrhotics (54\%), probably reflecting the high prevalence of H. pylori infection in the general Greek population [28]. We did not detect any association between $H$. pylori infection and the presence of gastroesophageal varices and/or PHG, reinforcing the impression that other mechanisms are involved in the pathogenesis of such complications of portal hypertension [27,29].

We did not find a significant association between the prevalence of peptic ulcer and the severity of cirrhosis, as expressed by Child-Pugh and MELD score or liver decompensation. Similar findings have also been demonstrated in most previous studies [5,22,30], while only Siringo et al reported a significant association between ascites and the presence of asymptomatic peptic ulcer [6].

The association between hepatic venous pressure gradient (HVPG) and peptic ulcer in cirrhotic patients has been 
controversial. Chen et al reported a significant association between HVPG levels higher than $12 \mathrm{mmHg}$ and the presence of peptic ulcer [5]. However, in another study by Bang et al such an association was not clearly documented [21]. In the most recent trial by Ardevol et al, 93\% of patients with peptic ulcer had HVPG $>10 \mathrm{mmHg}$, but HVPG was not measured in the entire study population [12]. We did not measure HVPG, but we tried to indirectly assess portal hypertension by liver elastography. According to our results, liver stiffness values were not associated with the presence of peptic ulcer. On the other hand, our cirrhotic patients, expected to have minimal probability for high-risk esophageal varices, based on low liver stiffness $(<20 \mathrm{kPa})$ and high platelet counts $\left(>150,000 / \mathrm{mm}^{3}\right)$, and therefore no need for upper GI screening endoscopy according to the Baveno VI consensus meeting, were found to have an increased prevalence $(23 \%)$ of peptic ulcer. Thus, the exclusion of the latter patient subgroup from screening upper GI endoscopy carries the risk of non-diagnosis of the usually asymptomatic peptic ulcer, precludes the early onset of treatment and eventually increases the risk of peptic ulcer complications such as bleeding. The exact pathophysiological basis of peptic ulcer development in cirrhosis has not been fully elucidated. H. pylori infection does not seem to be a driving factor, while, even if medications such as NSAIDs or antiplatelets strongly increase the risk of peptic ulcer in cirrhotic patients, their absence is not associated with a low risk of ulcerogenesis. Several pathogenetic factors have been implicated, such as impaired defensive mechanisms, decreased levels of the cytoprotective prostaglandin E or structural changes in the gastric mucosa [5]. Additionally, experimental studies have demonstrated increased gastric submucosal shunting of blood away from the gastric mucosa, leading to reduced oxygen supply, poor perfusion, and a lack of reactive response to toxic injury [5,31].

We did not observe a significant difference in the prevalence of peptic ulcer between sexes. This is in agreement with most studies, except for the study by Calvet et al which reported a male predominance among patients with peptic ulcer [22]. Unsurprisingly, most studies have shown that peptic ulcers are commonly observed in cirrhotic patients in the sixth decade of life $[6,7,23]$. Nevertheless, in accordance with our results, most studies did not find a difference in the mean age of cirrhotics with or without peptic ulcer. Finally, no difference across etiologies of liver disease was observed in our population. Similar findings have also been reported by most other studies, except for the study by Siringo et al, which found a higher prevalence of peptic ulcer in patients with cirrhosis due to chronic hepatitis B but provided no explanation [6].

In conclusion, our prospective study shows that the prevalence of peptic ulcer is high in asymptomatic patients with cirrhosis of various etiologies who undergo screening upper GI endoscopy. This is an interesting and potentially clinically relevant finding, as a prompt diagnosis of peptic ulcer will lead to appropriate management and potential prevention of serious complications in such a susceptible setting. One of the most interesting findings of our study was that the prevalence of peptic ulcer was higher in cirrhotic patients who may not be currently advised to have screening upper GI endoscopy, as they are considered to have a minimal probability of highrisk esophageal varices. These findings, of course, need to be investigated in larger prospective studies, as their confirmation could question the current recommendations for endoscopic screening in subgroups of cirrhotic patients and could support the need for endoscopic screening in the whole cirrhotic population. Finally, more studies are required to further clarify the underlying pathophysiologic mechanisms of ulcerogenesis in cirrhosis, the critical time point of its occurrence, the rate of possible recurrence, and the optimal prophylaxis for the elimination or minimization of the possibility of complications.

\section{Summary Box}

\section{What is already known:}

- Several studies have pointed out a higher prevalence of peptic ulcer disease in cirrhotics compared to non-cirrhotic patients

- Any episode of upper gastrointestinal (GI) bleeding due to peptic ulcer worsens the prognosis of cirrhotic patients

- The existing data have limitations, as many of the published studies were retrospective

- Thereareconflicting data concerning the contribution of Helicobacter pylori (H. pylori) infection and the potential role of portal hypertension severity

\section{What the new findings are:}

- We have documented a relatively high prevalence of peptic ulcer in asymptomatic patients with cirrhosis who underwent upper GI endoscopy for screening purposes

- H.pylori infection does not seem to play an important role in the pathophysiology of ulcerogenicity in patients with liver cirrhosis

- The prevalence of peptic ulcer was higher in patients who may not be currently advised to have screening upper GI endoscopy, as they are considered to have a minimal probability of high-risk esophageal varices

\section{References}

1. Bosch J, García-Pagán JC. Complications of cirrhosis. I. Portal hypertension. J Hepatol 2000;32:141-156.

2. D’Amico G, De Franchis R; Cooperative Study Group. Upper digestive bleeding in cirrhosis. Post-therapeutic outcome and prognostic indicators. Hepatology 2003;38:599-612.

3. de Franchis R; Baveno VI Faculty. Expanding consensus in portal hypertension: Report of the Baveno VI Consensus Workshop: Stratifying risk and individualizing care for portal hypertension. J Hepatol 2015;63:743-752.

4. Afessa B, Kubilis PS. Upper gastrointestinal bleeding in patients with hepatic cirrhosis: clinical course and mortality prediction. Am 
J Gastroenterol 2000;95:484-489.

5. Chen LS, Lin HC, Hwang SJ, Lee FY, Hou MC, Lee SD. Prevalence of gastric ulcer in cirrhotic patients and its relation to portal hypertension. J Gastroenterol Hepatol 1996;11:59-64.

6. Siringo S, Burroughs AK, Bolondi L, et al. Peptic ulcer and its course in cirrhosis: an endoscopic and clinical prospective study. J Hepatol 1995;22:633-641.

7. Zullo A, Rinaldi V, Meddi P, et al. Helicobacter pylori infection in dyspeptic cirrhotic patients. Hepatogastroenterology 1999;46:395-400.

8. Kirchner GI, Beil W, Bleck JS, Manns MP, Wagner S. Prevalence of Helicobacter pylori and occurrence of gastroduodenal lesions in patients with liver cirrhosis. Int J Clin Exp Med 2011;4:26-31.

9. Luo JC, Leu HB, Hou MC, et al. Cirrhotic patients at increased risk of peptic ulcer bleeding: a nationwide population-based cohort study. Aliment Pharmacol Ther 2012;36:542-550.

10. Leontiadis GI, Molloy-Bland M, Moayyedi P, Howden CW. Effect of comorbidity on mortality in patients with peptic ulcer bleeding: systematic review and meta-analysis. Am J Gastroenterol 2013;108:331-345.

11. Marmo R, Koch M, Cipolletta L, et al. Predictive factors of mortality from nonvariceal upper gastrointestinal hemorrhage: a multicenter study. Am J Gastroenterol 2008;103:1639-1647.

12. Ardevol A, Ibañez-Sanz G, Profitos J, et al. Survival of patients with cirrhosis and acute peptic ulcer bleeding compared with variceal bleeding using current first-line therapies. Hepatology 2018;67:1458-1471.

13. Holland-Bill L, Christiansen CF, Gammelager H, Mortensen RN, Pedersen L, Sørensen HT. Chronic liver disease and 90-day mortality in 21,359 patients following peptic ulcer bleeding-a Nationwide Cohort Study. Aliment Pharmacol Ther 2015;41:564-572.

14. Kitano S, Dolgor B. Does portal hypertension contribute to the pathogenesis of gastric ulcer associated with liver cirrhosis? $J$ Gastroenterol 2000;35:79-86.

15. Lo GH, Yu HC, Chan YC, et al. The effects of eradication of Helicobacter pylori on the recurrence of duodenal ulcers in patients with cirrhosis. Gastrointest Endosc 2005;62:350-356.

16. Pugh RN, Murray-Lyon IM, Dawson JL, Pietroni MC, Williams R. Transection of the oesophagus for bleeding oesophageal varices. $\mathrm{Br}$ J Surg 1973;60:646-649.

17. Berzigotti A. Non-invasive evaluation of portal hypertension using ultrasound elastography. J Hepatol 2017;67:399-411.

18. Roccarina D, Rosselli M, Genesca J, Tsochatzis EA. Elastography methods for the non-invasive assessment of portal hypertension.
Expert Rev Gastroenterol Hepatol 2018;12:155-164.

19. Spina GP, Arcidiacono R, Bosch J, et al. Gastric endoscopic features in portal hypertension: final report of a consensus conference, Milan, Italy, September 19, 1992. J Hepatol 1994;21:461-467.

20. Garcia-Tsao G, Sanyal AJ, Grace ND, Carey WD; Practice Parameters Committee of American College of Gastroenterology. Prevention and management of gastroesophageal varices and variceal hemorrhage in cirrhosis. Am J Gastroenterol 2007;102:2086-2102.

21. Bang CS, Baik GH, Kim JH, et al. Peptic ulcer disease in liver cirrhosis and chronic hepatitis: impact of portal hypertension. Scand J Gastroenterol 2014;49:1051-1057.

22. Calvet X, Navarro M, Gil M, et al. Epidemiology of peptic ulcer disease in cirrhotic patients: role of Helicobacter pylori infection. Am J Gastroenterol 1998;93:2501-2507.

23. Tzathas C, Triantafyllou K, Mallas E, Triantafyllou G, Ladas SD. Effect of Helicobacter pylori eradication and antisecretory maintenance therapy on peptic ulcer recurrence in cirrhotic patients: a prospective, cohort 2-year follow-up study. J Clin Gastroenterol 2008;42:744-749.

24. Cubillas R, Rockey DC. Portal hypertensive gastropathy: a review. Liver Int 2010;30:1094-1102.

25. Chang SS, Hu HY. Helicobacter pylori is not the predominant etiology for liver cirrhosis patients with peptic ulcer disease. Eur J Gastroenterol Hepatol 2013;25:159-165.

26. Wu CS, Lin CY, Liaw YF. Helicobacter pylori in cirrhotic patients with peptic ulcer disease: a prospective, case controlled study. Gastrointest Endosc 1995;42:424-427.

27. Chen JJ, Changchien CS, Tai DI, Chiou SS, Lee CM, Kuo CH. Role of Helicobacter pylori in cirrhotic patients with peptic ulcer. A serological study. Dig Dis Sci 1994;39:1565-1568.

28. Apostolopoulos P, Vafiadis-Zouboulis I, Tzivras M, Kourtessas D, Katsilambros N, Archimandritis A. Helicobacter pylori (H pylori) infection in Greece: the changing prevalence during a ten-year period and its antigenic profile. BMC Gastroenterol 2002;2:11.

29. Balzano A, Mosca S, Amitrano L, Di Blasi A, Piccirillo MM, Gigliotti T. Gastric antral erosions and Helicobacter pylori infection in cirrhotic patients: a pilot controlled study of oral bismuth vs ranitidine therapy. Ital J Gastroenterol 1991;23:132-135.

30. Chang SS, Hu HY. H. pylori eradication lower ulcers in cirrhosis. [Corrected]. J Dig Dis 2014;15:451-458.

31. Hashizume M, Tanaka K, Inokuchi K. Morphology of gastric microcirculation in cirrhosis. Hepatology 1983;3:1008-1012. 